\title{
Widespread occurrence of natural halogenated organics among temperate marine infauna
}

\author{
Kevin T. Fielman ${ }^{1, *}$, Sarah A. Woodin ${ }^{1,2}$, Michael D. Walla ${ }^{3}$, David E. Lincoln ${ }^{1}$ \\ ${ }^{1}$ Department of Biological Sciences, ${ }^{2}$ Marine Science Program, and ${ }^{3}$ Department of Chemistry, University of South Carolina, \\ Columbia, South Carolina 29208, USA
}

\begin{abstract}
Despite the global prevalence of marine sedimentary habitats, the potential for chemically mediated ecological interactions within these assemblages remains poorly known. Using GC/MS, we examined methanol extracts of worm, mollusc, and crustacean macroinfauna from 2 local intertidal sandflat communities for volatile organohalogens, a common, often bioactive class of secondary metabolites from marine organisms. Halogenated compounds were newly identified from 11 common polychaete species in the families Capitellidae, Chaetopteridae, Cirratulidae, Glyceridae, Pectinariidae and Spionidae. Tentative structures of these halometabolites are proposed based on GC/MS data. The represented chemical classes included aromatic mono- and dibrominated hydroxyphenylpropanoids, nitrogen-containing bromoalkylpyrroles, as well as brominated and chlorinated hydrocarbons and sulfides. Among the molluscs, only Terebra dislocata (Prosobranchia) contained an organohalogen compound (2,6-dibromophenol), which is likely derived from its diet of hemichordate worms. Volatile haloorganics were not found in amphipod and nemertean taxa. Possession of these compounds was not a function of any particular phylum, size categorization, or trophic mode, although hemichordates and all Capitellid and Spionid polychaetes collected at these sites invariably contained halocompounds. Most ( 40 of ca 54 taxa) of the numerically dominant macroinfaunal taxa at these 2 sites were examined in this survey; of these, $43 \%$ contained halometabolites, including the broadly distributed species Mediomastus ambiseta, Heteromastus filiformis, Streblospio benedicti, Tharyx marioni, and Saccoglossus kowalevskii. Thus, the potential for widespread occurrence of halogenated compounds among infauna is great. The presence of organohalogens in many common and abundant polychaete species suggests a general biological significance for these metabolites, a result of potential interest to marine ecologists, toxicologists and environmental chemists.
\end{abstract}

KEY WORDS: Marine natural product - Benthic - Hemichordate - Polychaete - Bromophenol - Bromopyrrole Haloaromatic Alkyl halide

\section{INTRODUCTION}

Sedimentary marine environments cover more than $70 \%$ of the earth's surface, yet our knowledge of the ecological importance of marine secondary metabolites from benthic animals is biased by the historical emphasis on tropical systems and their conspicuous epifaunal taxa (e.g. coelenterates, sponges, tunicates, etc.) (Paul 1992, Pawlik 1993, Hay 1996). For the more extensive temperate soft-bottom habitats, recent studies have demonstrated that some prevalent infaunal

\footnotetext{
·E-mail: fielman@biol.sc.edu
}

taxa contain and release high concentrations of halogenated aromatic compounds (reviewed by Woodin 1991, Steward et al. 1992). From an ecological perspective, infaunal chemistry may play an important structuring role in these assemblages by negatively affecting invertebrate larval recruitment and fish predation rates (Woodin 1991, Woodin \& Marinelli 1991 Woodin et al. 1993, Woodin et al. 1997, Woodin et al. unpubl.). Of the infauna examined in those investigations, all have been large (>3 cm length), predominantly perennial polychaete and hemichordate species; however, production or sequestration of brominated or chlorinated compounds by associated smaller, temporally variable organisms may confer tol- 
erance to contaminated habitats or similarly affect the ecological processes that structure sedimentary assemblages, thereby determining patterns of animal distribution and abundance in the field.

To evaluate the generality of these observations in our continuing investigation of these compounds' roles as determinants of benthic community structure and function, we asked whether possession of potentially bioactive halogenated compounds is a widespread phenomenon among the abundant, smaller macroinfauna found within a typical temperate benthic assemblage. This question was addressed by intensively sampling species from a representative soft-sediment intertidal habitat where polychaete and hemichordate worms, molluscs, and crustaceans occur. Our study documents the extraordinary prevalence of natural halogenated organic compounds among marine infauna, a character with potentially far-reaching ecological implications for benthic organisms from individual to community levels.

\section{MATERIALS AND METHODS}

Macroinfauna were collected in 1995 and 1996 from 2 intertidal sand flats in Debidue Creek, North Inlet, Georgetown, South Carolina $\left(33^{\circ} 20^{\prime} \mathrm{N}, 79^{\circ} 10^{\prime} \mathrm{W}\right)$ at the Belle W. Baruch Marine Laboratory. This estuary has been rated at $98 \%$ pristine quality and receives little anthropogenic input of halogenated organics (Blood \& Vernberg 1992). Animals were obtained either by hand digging or from $0.5 \mathrm{~mm}$-sieved sediments. At the field laboratory, organisms were removed from their tube or burrow structures if necessary, identified to the lowest taxonomic level possible and assigned to trophic categories given in Fauchald \& Jumars 1979, Taghon et al. 1980, and Ruppert \& Fox 1988. Based on an animal's size, each was placed into a minimal amount (150 $\mathrm{\mu l}$ to $20 \mathrm{ml})$ of GC/MS-grade methanol (Burdick \& Jackson, Muskegon, MI, USA). Small animals were extracted as pooled samples $(3$ to 10 individuals). Additional deuterium exchange experiments were conducted on animals extracted in acidified ethyl alcohol- $d_{6}(99 \%$ isotopic purity, Aldrich Chemical Co., Milwaukee, WI, USA) to aid in identification of functional groups containing labile hydrogen atoms (e.g. $\mathrm{OH}, \mathrm{NH}_{2}$ ) and to address potential methanol extraction artifacts. All samples were stored at $-20^{\circ} \mathrm{C}$ for a minimum of $1 \mathrm{wk}$ until analysis. Extracts for analysis consisted of the centrifuged (16000 RCF, $2 \mathrm{~min}$ ) supernatant from the solvent-soaked animal tissue; no grinding or other manipulation was performed.

Initial GC/MS analyses were made on a Hewlett Packard (Palo Alto, CA, USA) 5890 II gas chromatograph/5971 electron impact (EI) $(70 \mathrm{eV}$ ionization potential) mass spectrometer capable of unit mass resolution. This system was used to identify compounds containing halogen atoms, to obtain relative ion intensities from background-subtracted spectra, and to examine deuterium exchange products in a scan range from 50 to $350 \mathrm{mass} / \mathrm{charge}(\mathrm{m} / \mathrm{z})$. This instrument was also used to confirm the presence of low abundance molecular ions via selected ion monitoring (SIM). Volatile components from splitless injections $(2 \mu \mathrm{l})$ of the crude extracts were separated on a HP-5MS capillary column $(30 \mathrm{~m} \times 0.25 \mathrm{~mm}$ i.d., $0.25 \mu \mathrm{m}$ film thickness, Hewlett-Packard) using a constant $69 \mathrm{kPa}$ of helium. The GC thermal parameters were: injector port $275^{\circ} \mathrm{C}$; oven temperature program $70^{\circ} \mathrm{C}$ for $2 \mathrm{~min}$, $5^{\circ} \mathrm{C} \mathrm{min}^{-1}$ rise to $210^{\circ} \mathrm{C}, 10 \mathrm{~min}$ final hold; transfer line $280^{\circ} \mathrm{C}$. The $\mathrm{MS}$ ion source temperature was $180^{\circ} \mathrm{C}$. Compounds containing bromine or chlorine were recognized from the distinct isotopic signatures for the halogen atoms. Bromine occurs as 2 isotopes differing by 2 mass units (79/81) at nearly equal abundance (1:0.97). Similarly, chlorine occurs as 2 isotopes differing by 2 mass units $(35 / 37)$ at a $3: 1$ abundance ratio. Halogenated ions in a mass spectrum retain these ratios and are identified readily from these patterns. Within a taxon and among the volatile halogenated compounds identified, halocompound abundance was ranked by peak height in the total ion chromatogram (TIC).

Additional structural information and formulae for molecular and fragment ions were obtained using a HP 5890 II GC/VG 70SQ high resolution mass spectrometer (HRMS) system in EI mode $(70 \mathrm{eV})$ and perfluorokerosene calibrant. Scans were typically made over a range of 50 to $450 \mathrm{~m} / \mathrm{z}$. Splitless injections ( $2 \mu \mathrm{l}$ ) were separated on a Restek (Bellefonte, PA, USA) RT -5 capillary column $(30 \mathrm{~m} \times 0.25 \mathrm{~mm}$ i.d., $0.25 \mu \mathrm{m}$ film thickness). Variable GC thermal parameters were selected to optimize separations, but were typically: injector port $250^{\circ} \mathrm{C}$; oven temperature program $80^{\circ} \mathrm{C}$ for $1 \mathrm{~min}$, $5^{\circ} \mathrm{C} \mathrm{min} \mathrm{m}^{-1}$ rise to $210^{\circ} \mathrm{C}, 10^{\circ} \mathrm{C} \mathrm{min}-1$ rise to $300^{\circ} \mathrm{C}$, $10 \mathrm{~min}$ final hold. The transfer line and $\mathrm{MS}$ ion source temperatures were 250 and $200^{\circ} \mathrm{C}$, respectively. For several compounds we used high resolution (4000 to $15000)$ mass peak profiling with selected ion recording (MPPSIR) of $M, M+1$, and $M+2$ peaks to determine the composition of prominent ions in a manner similar to that of Grange \& Donnelly (1996).

Specific $\mathrm{m} / \mathrm{z}$ ratios selected from the range of molecular formulae suggested by isotopic abundances and high resolution mass determinations were monitored for maximized values across a mass peak profile. This technique provided narrowed potential molecular formulae ranges, or with sufficient resolution, unambiguous identification of molecular and fragment ion molecular weights. 


\section{RESULTS}

Brominated and chlorinated compounds were readily detected from 17 of $40(43 \%)$ macroinfaunal taxa examined at this site (Tables 1 \& 2). Although the fragment assignments and the structures presented from the mass spectral data herein are tentative and are intended to represent results consistent with the observed data and provide potential isomeric configurations only, aromatic bromohydroxyphenylpropan-

Table 1. Infaunal species from Debidue Creek containing volatile organohalogen compounds and their known or potential structures (within a species: ++ , abundant; + , present; $t$, trace; mix, mixture of related compounds). Compound letter designations correspond with those from the text and are listed in order of peak height. Compounds with the same molecular formulae but different letters are isomers. A ' $d$ ' indicates the position of a hydrogen atom detected by deuterium exchange. Trophic legend: FF, filter feeder; $\mathrm{H}$, herbivore; IF, interface feeder (sensu Taghon et al. 1980); P, predatory; SDF, surface deposit feeder; SSDF, subsurface deposit feeder. ${ }^{\alpha}$ Woodin et al. (1987); ${ }^{b}$ Fielman \& Targett (1995); ${ }^{c}$ Woodin (1991)

\begin{tabular}{|c|c|c|c|}
\hline Organism & Occurrence & Structure & \\
\hline \multicolumn{4}{|l|}{ POLYCHAETA } \\
\hline \multicolumn{4}{|l|}{ Capitellidae } \\
\hline Heteromastus filiformis (SSDF) & $D(++), C(+), A(t), B(t)$ & & \\
\hline Mediomastus ambiseta (SSDF) & $D(++), C(+), B(+), A(t)$ & & (B) $\mathrm{R1}=\mathrm{H}$; R2 = Br \\
\hline \multicolumn{4}{|r|}{$(\mathrm{C}, \mathrm{D}) \mathrm{R} 1=\mathrm{CHO}_{2} \mathrm{Me} ; \mathrm{R} 2=\mathrm{H}$} \\
\hline Chaetopterus variopedatus (FF) & $A(t)$ & & \\
\hline Spiochaetopterus oculatus (IF) & $A(t)$ & & \\
\hline \multicolumn{4}{|l|}{ Glyceridae } \\
\hline Glycera dibranchiata (P) & $A(t)$ & & \\
\hline \multicolumn{4}{|r|}{ E) $\mathrm{R} 1=\mathrm{C}_{7} \mathrm{H}_{15}, \mathrm{R} 2=\mathrm{H}$} \\
\hline \multirow{2}{*}{\multicolumn{4}{|c|}{$\begin{array}{l}(\mathrm{F}, \mathrm{G}) \mathrm{R} 1=\mathrm{C}_{8} \mathrm{H}_{17} ; \mathrm{R} 2=\mathrm{H} \\
\text { (H) } \mathrm{R} 1=\mathrm{C}_{7} \mathrm{H}_{15} ; \mathrm{R} 2=\mathrm{Br} \\
(\mathrm{l}, \mathrm{J}) \mathrm{R} 1=\mathrm{C}_{8} \mathrm{H}_{17} ; \mathrm{R} 2=\mathrm{Br}\end{array}$}} \\
\hline & & & \\
\hline \multicolumn{4}{|l|}{ POLYCHAETA } \\
\hline \multicolumn{4}{|l|}{ Pectinariidae } \\
\hline Cistenides gouldii (SSDF) & $\begin{array}{l}N(++), O(++), K(+) \\
L(t), M(t), P(t), Q(t)\end{array}$ & & $\begin{array}{ll}(\mathrm{K}-\mathrm{M}) \mathrm{R} 1=\mathrm{H} \\
\mathrm{S} \\
(\mathrm{N}, \mathrm{O}) \mathrm{R} 1=\mathrm{Cl} \\
(\mathrm{P}, \mathrm{Q}) \mathrm{R} 2=\mathrm{Br}\end{array}$ \\
\hline \multicolumn{4}{|l|}{ POLYCHAETA } \\
\hline \multicolumn{4}{|l|}{ Spionidae } \\
\hline Marenzellaria viridis (IF) & $\mathrm{AB}(+)$ & \multicolumn{2}{|r|}{$(\mathrm{R}-\mathrm{T}, \mathrm{V}) \mathrm{R} 1=\mathrm{Cl}$} \\
\hline Polydora socialis (SDF) & $\mathrm{R}(++), \mathrm{S}(\mathrm{t}), \mathrm{T}(\mathrm{t}), \mathrm{U}(\mathrm{t}), \mathrm{V}(\mathrm{t})$ & \multirow{2}{*}{\multicolumn{2}{|c|}{$(\mathrm{U}, \mathrm{W}, \mathrm{X}) \mathrm{R} 1=\mathrm{Br}$}} \\
\hline Scolelepis squamata (FF) & $\mathrm{AB}(+)$ & & \\
\hline Spiophanes bombyx (SDF) & $\mathrm{Y}(++), \mathrm{Z}(+), \mathrm{AB}(+), \mathrm{AA}(\mathrm{t})$ & \multirow{2}{*}{\multicolumn{2}{|c|}{$\begin{array}{l}\text { () } \mathrm{R} 1=\mathrm{H} ; \mathrm{R} 2=\mathrm{Cl} \\
\text { (Z) } \mathrm{R} 1=\mathrm{Cl} ; \mathrm{R} 2=\mathrm{CH}_{3} \\
\text { (AA) } \mathrm{R} 1=\mathrm{H} ; \mathrm{R} 2=\mathrm{Br}\end{array}$}} \\
\hline Streblospio benedicti (SDF) & $\begin{array}{l}S(++), T(+), V(+), W(+), \\
R(t), U(t), X(t)\end{array}$ & & \\
\hline \multicolumn{4}{|l|}{ HEMICHORDATA } \\
\hline Saccoglossus kowalevskii (SDF) & $\mathrm{AC}(++), \mathrm{AD}(\mathrm{t})$ & \multicolumn{2}{|r|}{$\begin{array}{l}\text { (AC) } \mathrm{R} 1=\mathrm{SO}_{3} \mathrm{Na} \\
\text { (AD) } \mathrm{R1}=\mathrm{H}\end{array}$} \\
\hline \multicolumn{4}{|l|}{ HEMICHORDATA } \\
\hline \multicolumn{4}{|l|}{ MOLLUSCA } \\
\hline \multicolumn{3}{|c|}{ (AE) $\mathrm{R} 1=\mathrm{R} 3=\mathrm{H}$; $\mathrm{R} 2=\mathrm{Br}$} & 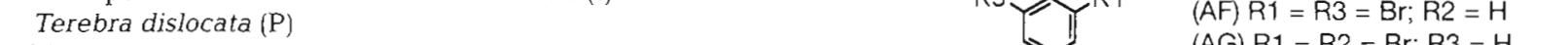 \\
\hline \multicolumn{4}{|r|}{$\begin{array}{l}\text { (AG) } \mathrm{R} 1=\mathrm{R} 2=\mathrm{Br} ; \mathrm{R} 3=\mathrm{H} \\
\text { (AH) } \mathrm{R} 1=\mathrm{R} 2=\mathrm{R} 3=\mathrm{Br}\end{array}$} \\
\hline \multicolumn{4}{|r|}{$(A-1) R 1=R 2=R 3=B r$} \\
\hline Arenicola brasiliensis (SSDF) ${ }^{d}$ & $\mathrm{AF}(++)$ & & \\
\hline \multicolumn{4}{|l|}{ Capitellidae } \\
\hline Notomastus lobatus (SSDF) ${ }^{c}$ & $\mathrm{AG}(++), \mathrm{AE}(+), \mathrm{AH}(+)$ & & \\
\hline \multicolumn{4}{|l|}{ Glyceridae } \\
\hline Glycera dibranchiata (P) & $\mathrm{AE}(\mathrm{t}, \mathrm{mix})$ & & \\
\hline
\end{tabular}


Table 2. Occurrence and absence of halogenated metabolites among infaunal trophic modes. Taxa containing halogenated compounds are marked with an asterix. Animals' feeding modes are based on categorizations from Fauchald \& Jumars (1979). Taghon et al. (1980), and Ruppert \& Fox (1988)

\begin{tabular}{|c|c|c|c|}
\hline SUB-SURFACE DEPOSIT & SURFACE DEPOSIT & INTERFACE & PREDATOR \\
\hline Hemichordata & Hemichordata & Polychaeta & Mollusca \\
\hline Balanoglossus aurantiacus ${ }^{*}$ & Saccoglossus kowalevski" & Chaetopteridae & Terebra dislocata ${ }^{\circ}$ \\
\hline Polychaeta & Mollusca & Spiochaetopterus oculatus ${ }^{*}$ & Nemertea \\
\hline Arenicolidae & Macomasp. & Spionidae & nemertean sp. 1 \\
\hline Arenicola brasiliensis" & Oligochaeta & Marenzellaria viridis ${ }^{\circ}$ & nemertean sp. 2 \\
\hline Capitellidae & oligochaete sp. & Scolelepis squamata & Polychaeta \\
\hline Heteromastus filiformis" & Polychaeta & & Glyceridae \\
\hline Mediomastus ambiseta" & Cirratulidae & & Glycera dibranchiata" \\
\hline Notomastus lobatus" & Tharyx marioni" & FILTER & Glycera americana \\
\hline Maldanidae & Flabellegeridae & Mollusca & Onuphidae \\
\hline Branchioasychis americana & Piromis eruca & Chione cancellata & Diopatra cuprea \\
\hline Clymenella torquata & Magelonidae & Mulinia lateralis & Kinbergonuphis jenneri \\
\hline Pectinariidae & Magelona sp. & Polychaeta & \\
\hline \multirow[t]{3}{*}{ Cistenides gouldi ${ }^{\circ}$} & Paraonidae & Chaetopteridae & \\
\hline & Aricidea fragilis & Chaetopterus variopedatus ${ }^{*}$ & UNKNOWN \\
\hline & Paraonis fulgens & & Crustacea \\
\hline HERBIVORE & Owenidae & & amphipod sp. \\
\hline Polychaeta & Owenia fusiformis & & Mollusca \\
\hline Ophelidae & Spionidae & & Acteocina canaliculata \\
\hline Armandia sp. & Polydora socialis. & & Polychaeta \\
\hline & Spiophanes bombyx" & & Arabellidae \\
\hline & Streblospio benedicti* & & Arabella iricolor \\
\hline & Terebellidae & & Drilonereis magna \\
\hline & Amphitrite ornata & & Lumbrineridae \\
\hline & & & Lumbrineris sp. \\
\hline
\end{tabular}

oid, bromophenol, bromopyrrole, as well as alkyl bromo- and chlorohydrocarbon and sulfide compound classes were evident from extracts of these

Table 3. Adult organism size and occurrence of volatile brominated or chlorinated compounds for the abundant species of Debidue Creek

\begin{tabular}{|c|c|c|c|c|c|c|}
\hline \multirow[t]{2}{*}{ Taxon } & \multirow[t]{2}{*}{ Species } & \multirow[t]{2}{*}{$\mathrm{Br} / \mathrm{Cl}$} & \multicolumn{4}{|c|}{ Adult length $(\mathrm{cm})$} \\
\hline & & & $<1$ & $1-3$ & $>3$ & $>>3$ \\
\hline \multicolumn{7}{|l|}{ POLYCHAETA } \\
\hline Arabellidae & Driloneris magna & & & & & $\mathrm{X}$ \\
\hline \multirow[t]{3}{*}{ Capitellidae } & Heteromastus filiformis & $\mathrm{x}$ & & $\mathrm{x}$ & & \\
\hline & Mediomastus ambiseta & $\mathrm{X}$ & $x$ & & & \\
\hline & Notomastus lobatus & $\mathrm{x}$ & & & & $\mathrm{X}$ \\
\hline Chaetopteridae & Spiochaetopterus oculatus & $x$ & & & $x$ & \\
\hline Cirratulidae & Tharyx marioni & $\mathrm{X}$ & $\mathrm{x}$ & & & \\
\hline \multirow[t]{2}{*}{ Onuphidae } & Diopatra cuprea & & & & & $x$ \\
\hline & Kinbergonuphis jenneri & & & & & $\mathrm{x}$ \\
\hline Paraonidae & Paraonis fu/gens & & $\mathrm{x}$ & & & \\
\hline Pectinaridae & Cistenides gouldii & $x$ & & $x$ & & \\
\hline \multirow[t]{3}{*}{ Spionidae } & Polydora sp. & $X$ & $\mathrm{x}$ & & & \\
\hline & Spiophanes bombyx & $X$ & & $x$ & & \\
\hline & Streblospio benedicti & $x$ & & $x$ & & \\
\hline Terebellidae & Amphitrite ornata & & & & $x$ & \\
\hline \multirow[t]{2}{*}{ HEMICHORDATA } & Balanoglossus aurantiacus & $\mathrm{X}$ & & & & $\mathrm{x}$ \\
\hline & Saccoglossus kowalevskii & $x$ & & & $x$ & \\
\hline \multirow[t]{2}{*}{ MOLLUSCA } & Macomasp. & & $x$ & & & \\
\hline & Terebra dislocata & $x$ & & $x$ & & \\
\hline
\end{tabular}

infaunal organisms. The largest, most abundant, haloorganic-containing species included the hemichordate worms Balanoglossus aurantiacus and Saccoglossus kowalevskij and the capitellid polychaete Notomastus lobatus, all known haloaromatic producers (Table 3). Many smaller, yet abundant species from the polychaete families Capitellidae, Chaetopteridae, Cirratulidae, Pectinariidae, and Spionidae also contained volatile haloorganic compounds. Possession of halogenated compounds was a particularly consistent character among the capitellids and spionids present at these study sites. Among the molluscs, only Terebra dislocata (Prosobranchia) had detectable levels of an organohalogen (2,6-dibromophenol) (Tables $1 \& 2$ ). Halometabolites were not detected in amphipod or nemertean species (Table 2). There were no interpretable relationships between the occurrence of haloorganics and an. organism's trophic mode (Table 2) or adult size (Table 3). 


\section{Capitellidae}

Four brominated aromatic metabolites (A-D) were identified from Heteromastus filiformis and Mediomastus ambiseta (Fig. 1a). Compound A, a bromovinylphenol, was also observed consistently from samples of Chaetopterus variopedatus, Spiochaetopterus occulatus, and Glycera dibranchiata, but not $G$. americana (Tabie 1). Compound B, a dibromovinylphenol, was characteristic of $M$. ambiseta but not $H$. filiformis. Compounds $C$ and $D$ were tentatively identified
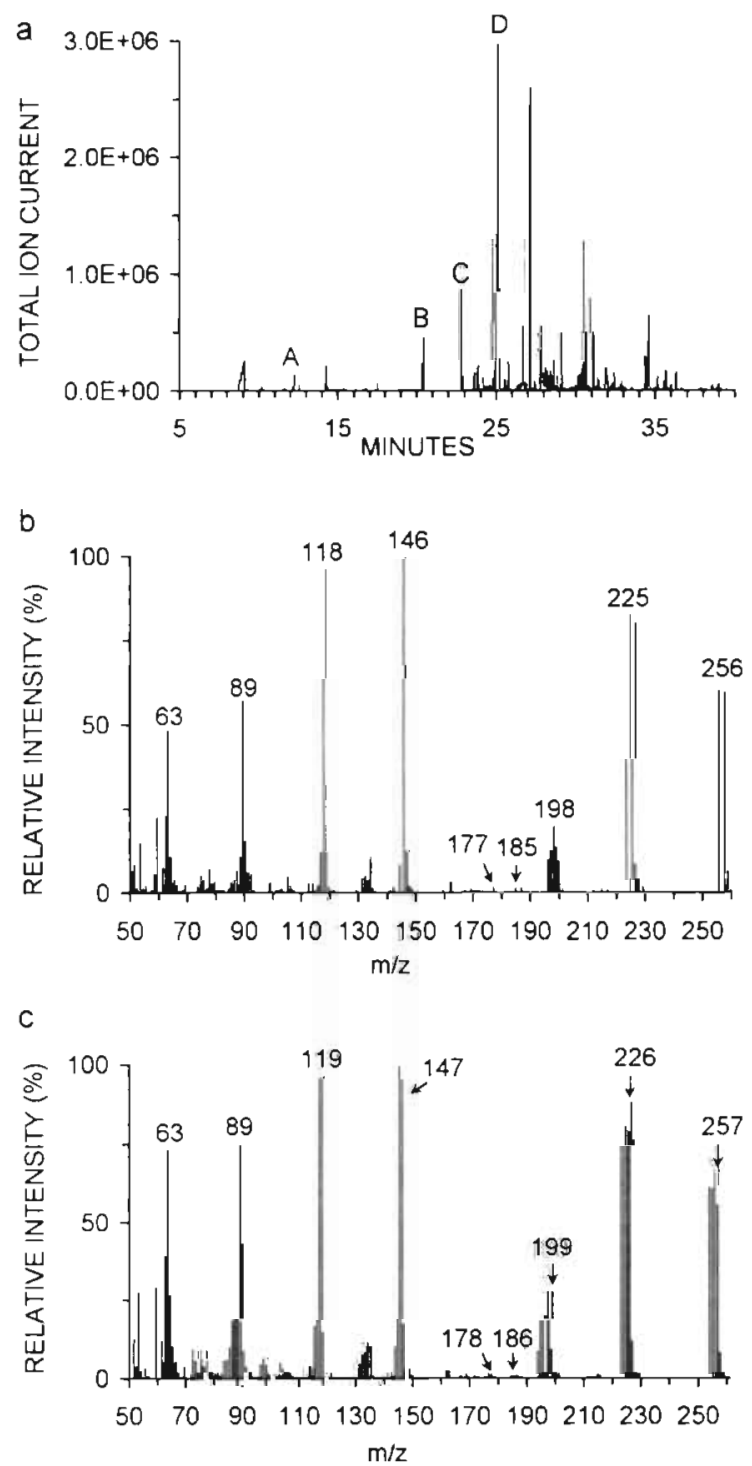

Fig. 1. Representative GC/MS data for selected halogenated, volatile compounds from Mediomastus ambiseta: (a) GC trace showing peaks for multiple brominated compounds (A-D). (b) Electron impact mass spectrum (EIMS) of compound D, a methyl ester of bromohydroxycinnamic acid. (c) EIMS for the partially deuterated form of compound D. See Table 1 for potential compound structures as bromohydroxycinnamic acid (= bromocoumarate) methyl ester isomers (Fig. 1b). The exact position of the ring substituents is currently not known. Thus, the structures presented in Table 1 represent plausible configurations subject to potential future revision. The mass spectral data for each compound are listed as the principal or diagnostic ions $(\mathrm{m} / \mathrm{z})$ with their intensities in relative percentage units in parentheses. Halogenated ion clusters are grouped and their ions are separated with a forward slash. The ions observed for each compound and their proposed elemental compositions were: (A) bromovinylphenol, $\mathrm{C}_{8} \mathrm{H}_{7} \mathrm{OBr}$, HRMS $\left({ }^{79} \mathrm{Br}\right)$ observed 197.9683, expected 197.9680: $\mathrm{M}^{+}$ $200(97) / 198(100), \quad\left(\mathrm{M}-\mathrm{C}_{2} \mathrm{H}_{2}\right)^{+} \quad 172(4) / 174(3), \quad(\mathrm{M}-\mathrm{Br})^{+}$ $119(25), 91(38), 90(19), 89(44), 63(26), 53(10)$; (B) dibromovinylphenol, $\mathrm{C}_{8} \mathrm{H}_{6} \mathrm{OBr}_{2}: \mathrm{M}^{+}$280(42)/278(86)/ 276(43), $(\mathrm{M}-\mathrm{Br})^{+}$199(35)/197(36), $\left(\mathrm{M}-\mathrm{Br}_{2}\right)^{+}$118(100), 89(43), 74(5),63(41), 59(14),53(14), 51(5), 50(6); and (C and D) bromohydroxycinnamic methyl ester, $\mathrm{C}_{10} \mathrm{H}_{9} \mathrm{O}_{3} \mathrm{Br}$, HRMS $\left({ }^{79} \mathrm{Br}\right)$ observed 255.9747 and 255.9765, respectively, expected 255.9735: $\mathrm{M}^{+} 258(68) /$ 256(69), $\left(\mathrm{M}-\mathrm{OCH}_{3}\right)^{+} 227(88) / 225(90),\left(\mathrm{M}-\mathrm{COOCH}_{3}\right)^{+}$ 198(20), $(\mathrm{M}-\mathrm{Br})^{+}$177(2), $\left(\mathrm{M}-\mathrm{CH}_{3} \mathrm{Br}\right)^{+}$162(3), (M$\left.\mathrm{CH}_{3} \mathrm{OBr}\right)^{+} 146(100), 134(10),\left(\mathrm{M}-\mathrm{CH}_{4} \mathrm{O}_{2} \mathrm{Br}\right)^{+}$118(92), 105(5), 89(50), 77(6), 75(3), 74(5), 63(41), 59(24), 53(13), $51(7), 50(6)$. Extraction in deuterated ethanol indicated an exchangeable phenolic hydrogen and no change in the ester functionality for this compound (Fig. 1c).

\section{Cirratulidae}

Tharyx marioni contained 3 alkylpyrroles (E-G) and their brominated derivatives $(\mathrm{H}-\mathrm{J})$ as major compounds of interest (Fig. 2a). Compound $\mathrm{H}$ was identified as the brominated form of $E$, a heptylpyrrole (Fig. 2b). Compounds $F$ and $G$ were identified as octylpyrrole isomers and compounds I and $\mathrm{J}$ as brominated octylpyrrole isomers. The exact position of the ring substituents and the presence of alkyl branching are currently not known. The mass spectral data for each compound are listed as the principal or diagnostic ions $(\mathrm{m} / \mathrm{z})$ with their intensities in relative percentage units in parentheses. Halogenated ion clusters are grouped and their ions are separated with a forward slash. The ions observed for each compound and their proposed elemental compositions were: (E) heptylpyrrole $\mathrm{C}_{11} \mathrm{H}_{19} \mathrm{~N}: \mathrm{M}^{+} 165(13),\left(\mathrm{M}-\mathrm{C}_{2} \mathrm{H}_{5}\right)^{+} 136(1),\left(\mathrm{M}-\mathrm{C}_{3} \mathrm{H}_{7}\right)^{+}$ 122(7), $\left(\mathrm{M}-\mathrm{C}_{4} \mathrm{H}_{9}\right)^{+}$108(1), $\left(\mathrm{M}-\mathrm{C}_{5} \mathrm{H}_{11}\right)^{+}$94(11), (M$\left.\mathrm{C}_{6} \mathrm{H}_{13}\right)^{+}$80(100), 67(3), 53(8); (F and G) octylpyrrole $\mathrm{C}_{12} \mathrm{H}_{21} \mathrm{~N}: \mathrm{M}^{+} 179(12),\left(\mathrm{M}-\mathrm{C}_{2} \mathrm{H}_{5}\right)^{+} 150(1),\left(\mathrm{M}-\mathrm{C}_{3} \mathrm{H}_{7}\right)^{+} 136$ (3), $\left(\mathrm{M}-\mathrm{C}_{4} \mathrm{H}_{9}\right)^{+}$122(3), $\left(\mathrm{M}-\mathrm{C}_{5} \mathrm{H}_{11}\right)^{+} 108(1),\left(\mathrm{M}-\mathrm{C}_{6} \mathrm{H}_{13}\right)^{+}$ 94(12), $\left(\mathrm{M}-\mathrm{C}_{7} \mathrm{H}_{15}\right)^{+}$80(100), 67(3), 53(8); (H) bromoheptylpyrrole $\mathrm{C}_{11} \mathrm{H}_{18} \mathrm{NBr}: \mathrm{M}^{+} 245(11) / 243(11),(\mathrm{M}-\mathrm{Br})^{+}$ 164(23), $\left(\mathrm{M}-\mathrm{C}_{6} \mathrm{H}_{13}\right)^{+}$160(96)/158(100), 147(1), 145(1), 


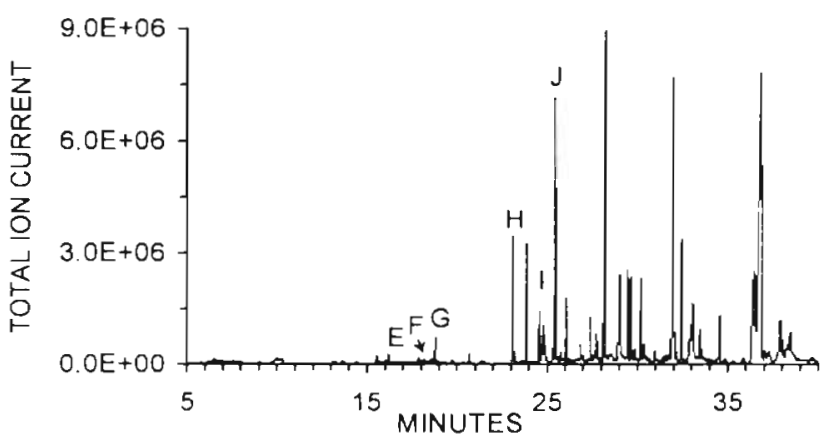

b

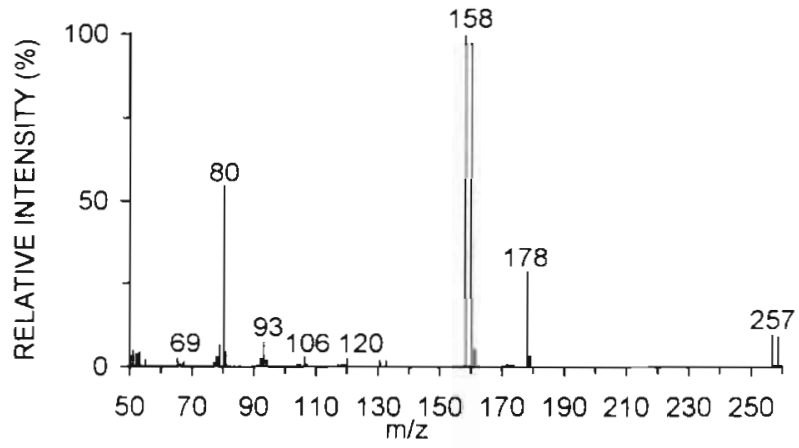

c

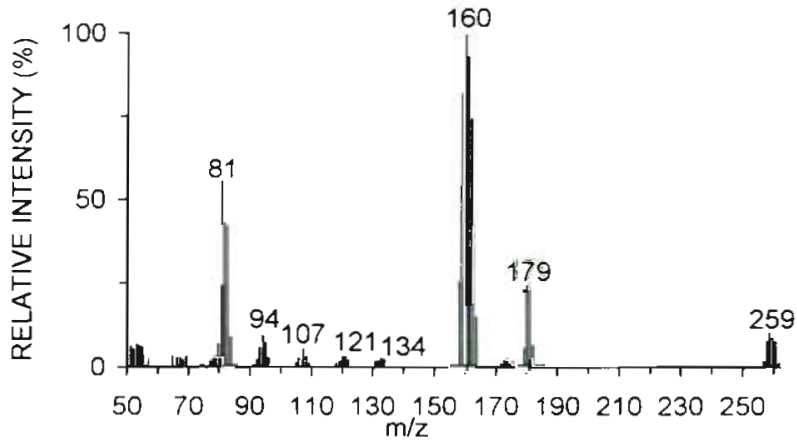

Fig. 2. Representative GC/MS data for selected volatile compounds of interest from Tharyx marioni. (a) GC trace showing peaks for presumed precursors $(E-G)$ and their brominated analogues $(\mathrm{H}-\mathrm{J})$. (b) Electron impact mass spectrum (EIMS) of compound I, a bromoalkylpyrrole. (c) EIMS for the partially deuterated form of 1 . See Table 1 for potential compound structures

133(3), 131(3), $\left(\mathrm{M}-\mathrm{C}_{3} \mathrm{H}_{8} \mathrm{Br}\right)^{+}$120(2), $\left(\mathrm{M}-\mathrm{C}_{4} \mathrm{H}_{10} \mathrm{Br}\right)^{+} 106$ (3), $\left(\mathrm{M}-\mathrm{C}_{5} \mathrm{H}_{11} \mathrm{Br}\right)^{+}$93(8), $\left(\mathrm{M}-\mathrm{C}_{6} \mathrm{H}_{12} \mathrm{Br}\right)^{+}$80(50), 65(4), $55(3), 51(7) ;$ (I and J) bromooctylpyrrole $\mathrm{C}_{12} \mathrm{H}_{20} \mathrm{NBr}$, HRMS $\left({ }^{79} \mathrm{Br}\right)$ observed 257.0731 and 257.0746 , respectively, expected 257.0779: $\mathrm{M}^{+} 259(10) / 257(10),(\mathrm{M}-\mathrm{Br})^{+}$ 178(29), $\left(\mathrm{M}-\mathrm{C}_{7} \mathrm{H}_{15}\right)^{+} 160(99) / 158(100), 147(1), 145(1)$, 133(3), 131(3), $\left(\mathrm{M}-\mathrm{C}_{4} \mathrm{H}_{10} \mathrm{Br}\right)^{+}$120(3), $\quad\left(\mathrm{M}-\mathrm{C}_{5} \mathrm{H}_{12} \mathrm{Br}\right)^{+}$ 106(4), $\left(\mathrm{M}-\mathrm{C}_{6} \mathrm{H}_{13} \mathrm{Br}\right)^{+}$93(8), $\left(\mathrm{M}-\mathrm{C}_{7} \mathrm{H}_{1.4} \mathrm{Br}\right)^{+} 80(56), 65(3)$, $55(3), 51(5)$. Extraction in acidified ethanol- $d_{6}$ indicated one exchangeable hydrogen on the pyrrole nitrogen and a second, likely transferred to the $\alpha$-car- bon of the pyrrole ring under our highly acidic extraction conditions (Fig. 2c) (Chadwick 1990).

\section{Pectinariidae}

Chlorosulfide ( $N$ and $O$ ) and bromosulfide ( $P$ and $Q$ ) isomer pairs and their presumed precursor molecules $(\mathrm{K}-\mathrm{M})$ were the abundant compounds of interest detected in Cistenides gouldii extracts (Fig. 3a). The methyl sulfide structure was inferred from the consistent loss of $\mathrm{m} / \mathrm{z}, 48$ from the molecular ion $\left(\mathrm{M}-\mathrm{CH}_{4} \mathrm{~S}\right)^{+}$ and high resolution isotopic abundance measurements suggesting the presence of sulfur. For the remainder of the molecule, branch position and length cannot be assigned unambiguously by mass spectrometry. Mass peak profiling by selected ion recording (MPPSIR) at a resolution of 6000 was used to narrow the range of molecular weights for compound $\mathrm{K}$ to between 186.1300 and 186.1550, consistent with our favored formula of $\mathrm{C}_{11} \mathrm{H}_{22} \mathrm{~S}$ (expected 186.1442). Data collected

a

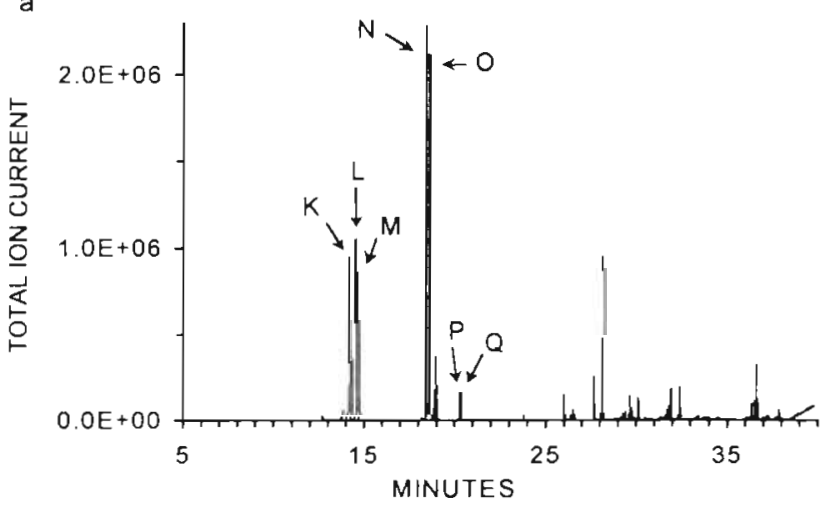

b

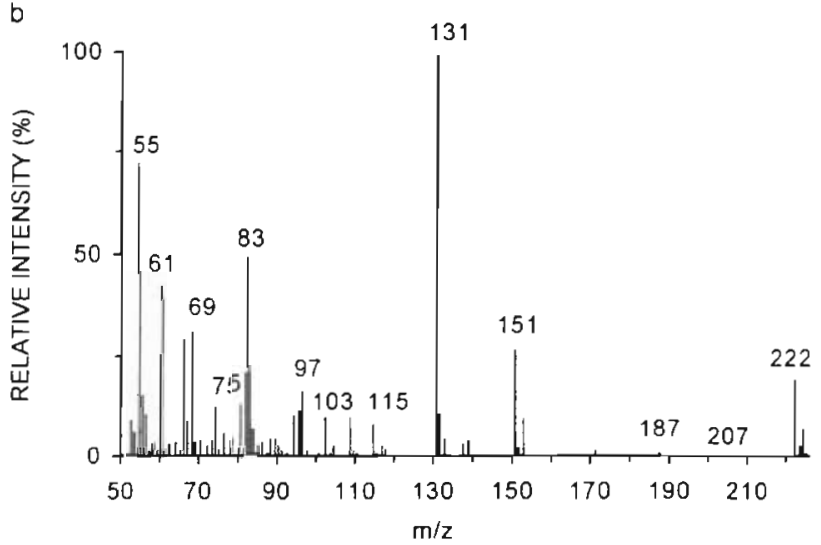

Fig. 3. Representative GC/MS data for selected volatile compounds of interest from Cistenides gouldii. (a) GC trace showing presumed precursors $(\mathrm{K}-\mathrm{M})$ and their chlorinated $(\mathrm{N}, \mathrm{O})$ and brominated ( $P, Q$ ) analogues. (b) Electron impact mass spectrum of compound $N_{1}$ a chlorosulfide. See Table 1 for potential com-pound structures 
by MPPSIR were also consistent with our measured mass for chlorinated compound $\mathrm{N}, \mathrm{C}_{11} \mathrm{H}_{21} \mathrm{SCl}$ (observed 222.1191, expected 222.1202), and favored formula for brominated compound $\mathrm{P}$ of $\mathrm{C}_{11} \mathrm{H}_{21} \mathrm{SBr}$ (expected 266.0704). The mass spectral data for each compound are listed as the principal or diagnostic ions $(\mathrm{m} / \mathrm{z})$ with their intensities in relative percentage units in parentheses. Halogenated ion clusters are grouped and their ions are separated with a forward slash. The ions observed for each compound and their proposed elemental compositions were: $(\mathrm{K}) \mathrm{C}_{11} \mathrm{H}_{22} \mathrm{~S}: \mathrm{M}^{+} 186(5)$, $\left(\mathrm{M}-\mathrm{CH}_{3}\right)^{+}$171(6), $\left(\mathrm{M}-\mathrm{C}_{2} \mathrm{H}_{5}\right)^{+} 157(3),\left(\mathrm{M}-\mathrm{CH}_{4} \mathrm{~S}\right)^{+} 138(31)$, $\left(\mathrm{M}-\mathrm{C}_{4} \mathrm{H}_{7}\right)^{+}$131(81), $\left(\mathrm{M}-\mathrm{C}_{5} \mathrm{H}_{11}\right)^{+}$115(3), $\left(\mathrm{M}-\mathrm{C}_{3} \mathrm{H}_{9} \mathrm{~S}\right)^{+} 109$ (2), $\left(\mathrm{M}-\mathrm{C}_{4} \mathrm{H}_{9} \mathrm{~S}\right)^{+}$97(22), 87(13), 83(80), 81(12), 79(8), 75(9), 69(54), 67(32), 61(72), 57(9), 55(100), 53(13); (L) $\mathrm{C}_{11} \mathrm{H}_{22} \mathrm{~S}: \mathrm{M}^{+}$186(2), (M-CH$)^{+} 171(7),\left(\mathrm{M}-\mathrm{CH}_{4} \mathrm{~S}\right)^{+}$ 138(2), $\left(\mathrm{M}-\mathrm{C}_{3} \mathrm{H}_{6} \mathrm{~S}\right)^{+}$109(1), 95(2), $\left(\mathrm{M}-\mathrm{C}_{7} \mathrm{H}_{13}\right)^{+}$89(100), 83(2), 77(2), 67(7), 61(13), 55(11); (M) $\mathrm{C}_{11} \mathrm{H}_{22} \mathrm{~S}: \mathrm{M}^{+}$ 186(2), $\left(\mathrm{M}-\mathrm{CH}_{3}\right)^{+} 171(9),\left(\mathrm{M}-\mathrm{CH}_{4} \mathrm{~S}\right)^{+} 138(2),\left(\mathrm{M}-\mathrm{C}_{4} \mathrm{H}_{7}\right)^{+}$ 131(16), $\left(\mathrm{M}-\mathrm{C}_{3} \mathrm{H}_{6} \mathrm{~S}\right)^{+}$109(2), 95(3), $\left(\mathrm{M}-\mathrm{C}_{7} \mathrm{H}_{13}\right)^{+} 89(100)$, $83(10), 79(3), 77(2), 75(2), 74(2), 73(2), 67(13), 61(26)$, 55(22), 53(5); (N) (Fig. 3b) $\mathrm{C}_{11} \mathrm{H}_{21} \mathrm{SCl}: \mathrm{M}^{+}$224(7)/

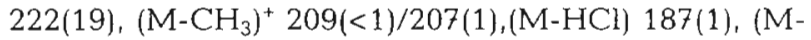
$\left.\mathrm{CH}_{4} \mathrm{~S}\right)^{+} \quad 176(<1) / 174(1),\left(\mathrm{M}-\mathrm{C}_{5} \mathrm{H}_{11}\right)^{+} \quad 153(10) / 151(26)$. $\left(\mathrm{M}-\mathrm{CH}_{4} \mathrm{SCl}\right)^{+} 138(4), 137(4),\left(\mathrm{M}-\mathrm{C}_{4} \mathrm{H}_{7} \mathrm{Cl}\right)^{+}$131(100), (M$\left.\mathrm{C}_{5} \mathrm{H}_{11} \mathrm{Cl}\right)^{+}$115(8), (M- $\left.\mathrm{C}_{3} \mathrm{H}_{9} \mathrm{SCl}\right)^{+}$109(10), 103(10), (M$\left.\mathrm{C}_{4} \mathrm{H}_{9} \mathrm{SCl}\right)^{+} 97(17), 83(50), 75(13), 69(31), 67(29), 61(41)$, 55(70); (O) $\mathrm{C}_{11} \mathrm{H}_{21} \mathrm{SCl}: \mathrm{M}^{+} 224(3) / 222(8),\left(\mathrm{M}-\mathrm{C}_{2} \mathrm{H}_{5}\right)^{+} 195$ $(1) / 193(4), 187(1), 171(7), 157(2), 139(4), 138(4), 131$ (<1), 115(1), 109(10), 103(2), 97(9), 89(100), 83(24), 75 (7), 69(15), 67(15), 61(18), 55(34), 53(6); (P) $\mathrm{C}_{11} \mathrm{H}_{21} \mathrm{SBr}$ : $\mathrm{M}^{+}$268(5)/266(6), 197(3)/195(3), 187(13), 139(18), 131 (28), 115(7), 109(3), 97(37), 89(5), 83(100), 75(3), 69(40), 67(27), 61(37), 57(22), 55(70); (Q) $\mathrm{C}_{11} \mathrm{H}_{21} \mathrm{SBr}: \mathrm{M}^{+} 268(5) /$ $266(6), 187(36), 139(25), 131(3), 109(8), 97(45), 89(80)$, $83(100), 75(6), 69(56), 67(22), 61(26), 57(21), 55(78)$, $53(10)$.

\section{Spionidae}

Members of the 5 different spionid genera examined contained halogenated compounds (Table 1). Streblospio benedicti, the most common and abundant spionid at our collection sites, typically contained 4 chlorinated $(\mathrm{R}-\mathrm{T}, \mathrm{V})$ and 3 brominated $(\mathrm{U}, \mathrm{W}, \mathrm{X})$ branched alkylhalides (Fig. 4a). Polydora socialis contained a subset of these compounds $(\mathrm{R}-\mathrm{V})$. Molecular formulae are those suggested by MPPSIR. Branch position and length cannot be assigned unambiguously by mass spectrometry and the structure presented in Table 1 is intended to represent this general compound class. The mass spectral data for each compound are listed as the principal or diagnostic ions $(\mathrm{m} / \mathrm{z})$ with their intensities in relative percentage units in parentheses. Halo-
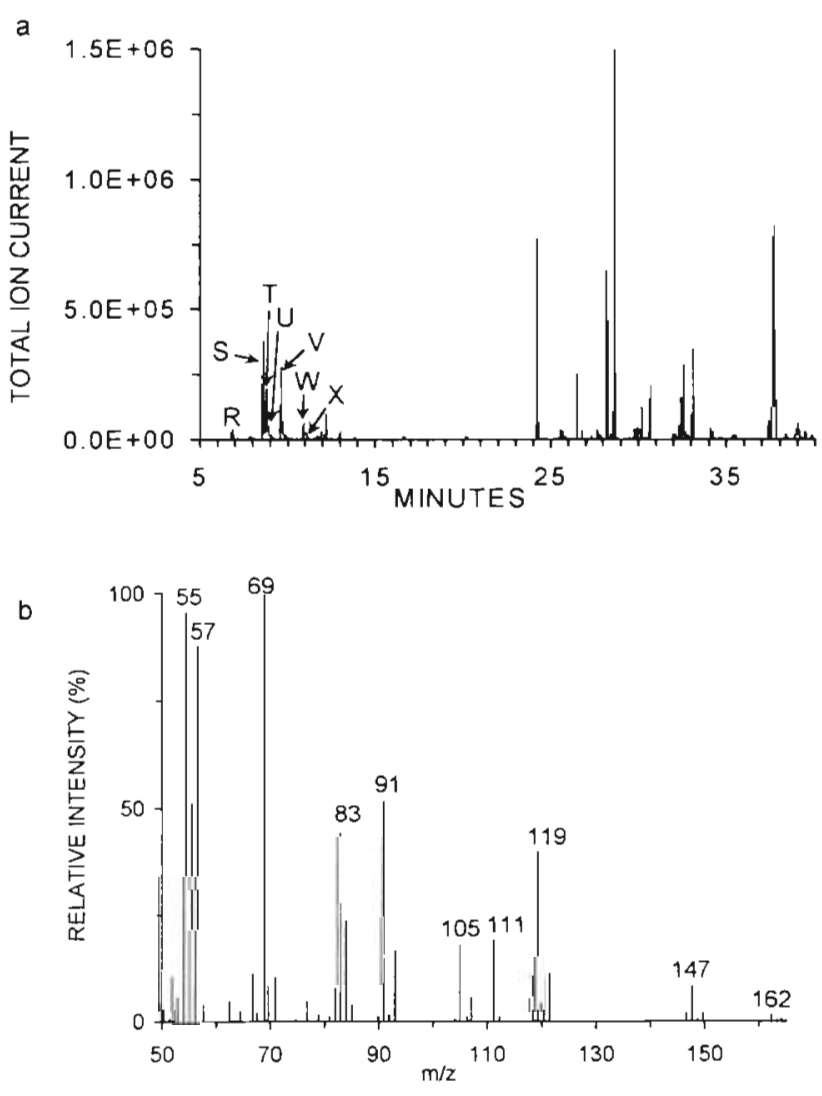

Fig, 4. Representative GC/MS data for selected halogenated volatile compounds from Streblospio benedicti. (a) GC trace showing peaks for chlorinated (R-U) and brominated (V,W) compounds. (b) Electron impact mass spectrum of compound $\mathrm{S}$, a chlorinated hydrocarbon. See Table 1 for potential compound structures

genated ion clusters are grouped and their ions are separated with a forward slash. The ions observed for each compound and their proposed elemental compositions were: (R) $\mathrm{C}_{9} \mathrm{H}_{19} \mathrm{Cl}: \mathrm{M}^{+}$not observed, $\left({\left.\mathrm{M}-\mathrm{CH}_{3}\right)^{+}}^{+}\right.$ 149(3)/147(9), $\left(\mathrm{M}-\mathrm{C}_{3} \mathrm{H}_{7}\right)^{+}$121(12)/119(40), 111(20), (M$\left.\mathrm{C}_{4} \mathrm{H}_{9}\right)^{+} 107(6) / 105(18), 91(52), 84(24), 83(45), 77(5), 71$ (11), 69(100), 67(12), 63(6), 57(88), 56(52), 55(95), 53 (12), 51(5); (S) (Fig. 4 b) $\mathrm{C}_{9} \mathrm{H}_{19} \mathrm{Cl}: \mathrm{M}^{+} 164(<1) / 162(1)$, $\left(\mathrm{M}-\mathrm{CH}_{3}\right)^{+} 149(6) / 147(20),\left(\mathrm{M}-\mathrm{C}_{3} \mathrm{H}_{7}\right)^{+}$121(13)/119(43), 111(16), $\left(\mathrm{M}-\mathrm{C}_{4} \mathrm{H}_{9}\right)^{+}$107(6)/105(17), 93(16), 91(49), 84 (29), 83(41), 77(5), 71(12), 69(91), 67(8), 63(5), 57(100), $56(52), 55(90), 53(9), 51(2) ;(\mathrm{T}) \mathrm{C}_{9} \mathrm{H}_{19} \mathrm{Cl}: \mathrm{M}^{+}$not observed, $\left(\mathrm{M}-\mathrm{CH}_{3}\right)^{+} 149(<1) / 147(1),\left(\mathrm{M}-\mathrm{C}_{2} \mathrm{H}_{5}\right)^{+} 135(8) /$ 133(25), $\left(\mathrm{M}-\mathrm{C}_{4} \mathrm{H}_{9}\right)^{+}$107(6)/105(20), 97(46), 93(3), 91(9), 83(2), 77(2), 71(7), 70(7), 69(21), 57(100), 56(60), 55(74), 53(4); (U) $\mathrm{C}_{9} \mathrm{H}_{19} \mathrm{Br}: \mathrm{M}^{+}$not observed, $\left(\mathrm{M}-\mathrm{C}_{4} \mathrm{H}_{9}\right)^{+} 151$ (11)/149(10), $\left(\mathrm{M}-\mathrm{C}_{5} \mathrm{H}_{11}\right)^{+} 137(95) / 135(96),\left(\mathrm{M}-\mathrm{C}_{7} \mathrm{H}_{15}\right)^{+}$ 109(7)/107(6), 97(8), 85(5), 84(8), 83(18), 82(10), 72(6), $71(68), 70(23), 69(44), 67(10), 58(48), 57(100), 56(52)$, 55(97); (V) $\mathrm{C}_{9} \mathrm{H}_{19} \mathrm{Cl}: \mathrm{M}^{+}$not observed, $\left(\mathrm{M}-\mathrm{C}_{4} \mathrm{H}_{9}\right)^{+} 107$ $(5) / 105(16), 97(7), 93(30), 91(100), 85(5), 84(5), 83(11)$, $77(2), 71(12), 70(11), 69(27), 57(32), 56(18), 55(50)$; 
(W) $\mathrm{C}_{9} \mathrm{H}_{19} \mathrm{Br}: \mathrm{M}^{+} 208(2) / 206(3),\left(\mathrm{M}-\mathrm{CH}_{3}\right)^{+}$193(3)/191 (3), $\left(\mathrm{M}-\mathrm{C}_{3} \mathrm{H}_{7}\right)^{+} 165(37) / 163(40),\left(\mathrm{M}-\mathrm{C}_{4} \mathrm{H}_{9}\right)^{+} 151(11) /$ 149(10), $\left(\mathrm{M}-\mathrm{C}_{5} \mathrm{H}_{11}\right)^{+} 137(67) / 135(69),(\mathrm{M}-\mathrm{Br})^{+} 129(<1) /$ 127(1), $\left(\mathrm{M}-\mathrm{C}_{6} \mathrm{H}_{13}\right)^{+}$123(3)/121(3), 111(15), 85(26), $84(19), 83(52), 71(43), 70(11), 69(86), 57(95), 56(54)$, 55(100); (X) $\mathrm{C}_{9} \mathrm{H}_{20} \mathrm{Br}: \mathrm{M}^{+} 209(<1) / 207(1), \quad\left(\mathrm{M}-\mathrm{C}_{2} \mathrm{H}_{6}\right)^{+}$ $179(8) / 177(8),\left(\mathrm{M}-\mathrm{C}_{4} \mathrm{H}_{10}\right)^{+} 151(43) / 149(45),\left(\mathrm{M}-\mathrm{C}_{5} \mathrm{H}_{12}\right)^{+}$ $137(8) / 135(9),\left(\mathrm{M}-\mathrm{C}_{6} \mathrm{H}_{14}\right)^{+}$123(3)/121(3), 111(2), $97(50)$, $83(3), 71(7), 70(9), 69(30), 57(100), 56(42), 55(76), 53(6)$.

Spiophanes bombyx contained a mixture of 3 chlorinated $(Y, Z, A B)$ and 1 brominated alkylhalide $(A A)$ of variable chain lengths and unsaturation (Fig. 5). Marenzellaria viridis and Scolelepis squamata contained just one of these compounds $(A B)$ in abundance (see Fig. 5b for mass spectrum). Double bond and bromine positions cannot be assigned unambiguously from our present mass spectra, although the spectrum for compound $\mathrm{AA}$ is a good match for the known spectrum of 1-bromo-5-decene. Thus, these compounds remain unnamed and the structures proposed in Table 1 should be considered hypotheses. The mass spectral data for each compound are listed as the principal or diagnostic ions $(\mathrm{m} / \mathrm{z})$ with their intensities in relative
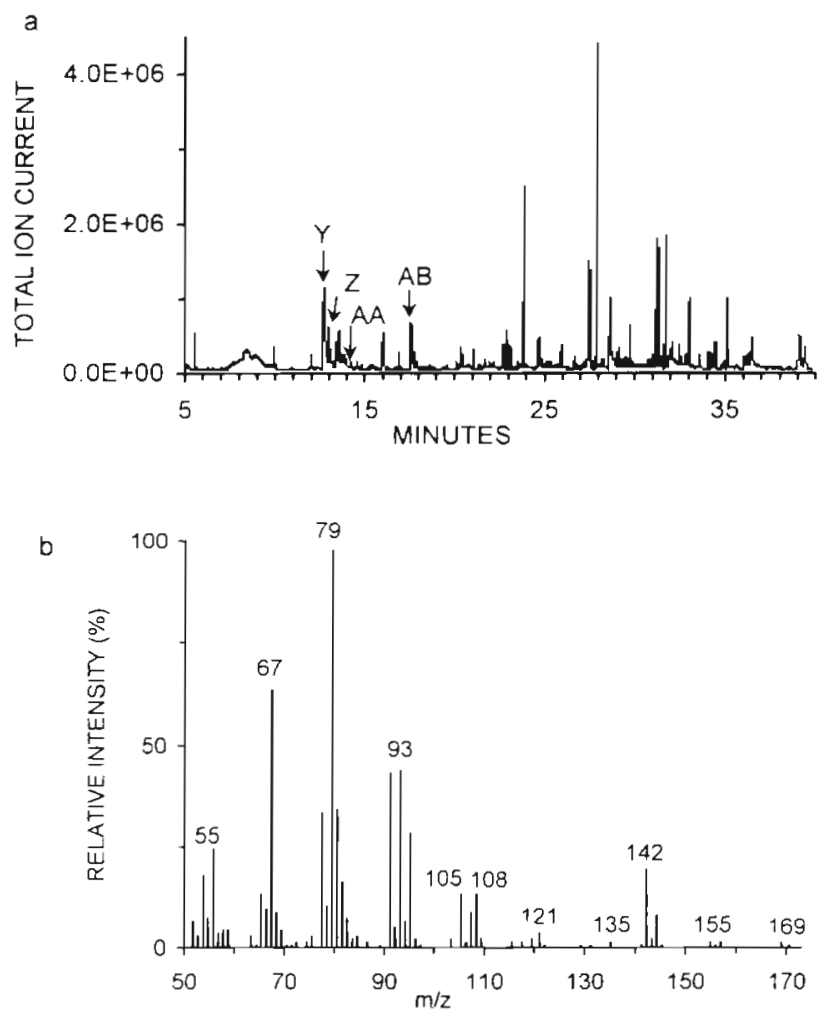

Fig. 5. Representative GC/MS data for selected halogenated volatile compounds from Spiophanes bombyx. (a) GC trace showing peaks for chlorinated ( $Y, Z, A B$ ) and brominated $(Z)$ compounds. (b) Electron impact mass spectrum of compound $A B$, a chlorinated, unsaturated hydrocarbon. See Table 1 for potential compound structures percentage units in parentheses. Halogenated ion clusters are grouped and their ions are separated with a forward slash. The ions observed for each and their proposed elemental compositions were: ( $\mathrm{Y}$ ) $\mathrm{C}_{10} \mathrm{H}_{19} \mathrm{Cl}$ $\mathrm{M}^{+}$176(4)/174(12), $\left(\mathrm{M}-\mathrm{C}_{2} \mathrm{H}_{4}\right)^{+} \quad 148(<1) / 146(1), \quad(\mathrm{M}-$ $\left.\mathrm{C}_{3} \mathrm{H}_{6}\right)^{+} 134(<1) / 132(1),\left(\mathrm{M}-\mathrm{C}_{4} \mathrm{H}_{8}\right)^{+}$120(2)/118(5), (M$\left.\mathrm{C}_{2} \mathrm{H}_{6} \mathrm{Cl}\right)$ 109(11), $\left(\mathrm{M}-\mathrm{C}_{5} \mathrm{H}_{10}\right)^{+}$106(6)/104(17), 98(2), 97 $(12), 96(6), 95(17), 84(12), 83(20), 82(16), 81(23), 70(26)$ $69(40), 68(23), 67(45), 58(13), 57(10), 56(40), 55(100)$ 54(22), 53(18); (Z) $\mathrm{C}_{11} \mathrm{H}_{21} \mathrm{Cl}: \mathrm{M}^{+} 190(5) / 188(13),(\mathrm{M}$ $\left.\mathrm{C}_{4} \mathrm{H}_{8}\right)^{+} \quad 134(<1) / 132(3), \quad\left(\mathrm{M}-\mathrm{C}_{5} \mathrm{H}_{10}\right)^{+} 120(15) / 118(44)$ $\left(\mathrm{M}-\mathrm{C}_{3} \mathrm{H}_{6} \mathrm{Cl}\right) 111(7), 97(4), 95(10), 84(11), 83(25), 82(7)$ $81(23), 79(14), 77(9), 70(25), 69(58), 68(16), 67(31)$ $58(24), 57(20), 56(100), 55(78), 53(22) ;(\mathrm{AA}) \mathrm{C}_{10} \mathrm{H}_{19} \mathrm{Br}$ $\mathrm{M}^{+} 220(5) / 218(6),\left(\mathrm{M}-\mathrm{C}_{4} \mathrm{H}_{3}\right)^{+} 164(6) / 162(6),\left(\mathrm{M}-\mathrm{C}_{5} \mathrm{H}_{10}\right)^{+}$ $150(13) / 148(14), \quad\left(\mathrm{M}-\mathrm{C}_{6} \mathrm{H}_{11}\right)^{+} 137(7) / 135(9), \quad\left(\mathrm{C}_{2} \mathrm{H}_{6} \mathrm{Br}\right)^{+}$ $109(10), 97(27), 95(17), 84(22), 83(39), 82(17), 81(19)$ $70(21), 69(53), 68(13), 67(38), 58(71), 57(16), 56(30)$, $55(100), 54(22), 53(21)_{i}(\mathrm{AB}) \mathrm{C}_{12} \mathrm{H}_{19} \mathrm{Cl}: \mathrm{M}^{+}$not observed, $\left(\mathrm{M}-\mathrm{C}_{2} \mathrm{H}_{5}\right)^{+} 171(1) / 169(2),\left(\mathrm{M}-\mathrm{C}_{3} \mathrm{H}_{7}\right)^{+} 157(2) / 155(3),(\mathrm{M}-$ $\left.\mathrm{C}_{4} \mathrm{H}_{8}\right)^{+}$144(9)/142(20), $\left(\mathrm{M}-\mathrm{C}_{5} \mathrm{H}_{9}\right)^{+}$131(1)/129(2), (M$\left.\mathrm{C}_{4} \mathrm{H}_{7} \mathrm{Cl}\right)^{+}$108(14), $\left(\mathrm{M}-\mathrm{C}_{7} \mathrm{H}_{9}\right)^{+} 107(9) / 105(14), 95(30)$ $93(45), 91(44), 81(17), 80(35), 79(100), 78(11), 77(34)$, $67(64), 65(14), 55(24), 53(17)$. The parent and largest fragment ions $(\mathrm{M}-\mathrm{CH} 3)^{+}$for compound $\mathrm{AB}$ were observed by SIM to be $200 / 198$ and $183 / 185$, respectively.

\section{DISCUSSION}

One common attribute of secondary metabolites from marine organisms is the presence of chlorine, bromine, or iodine. Our study examined marine infauna for low molecular weight, volatile haloorganic compounds. Using a single analytical technique (GC/MS), we readily determined that a surprising proportion $(43 \%)$ of common macrobenthic taxa from a widespread temperate sedimentary habitat contain naturally occurring, volatile haloorganics from diverse chemical classes (bromohydroxyphenylpropanoids, bromophenols, bromopyrroles, brominated and chlorinated sulfides and hydrocarbons). This novel result broadens the distribution of halometabolites, particularly within the Polychaeta, and raises numerous questions regarding the origin of these metabolites and their potential biological roles that we address below. In the interpretation of these results, note that assignment of definitive isomeric structures from mass spectral data is difficult, if not impossible, for many compounds, particularly unknowns separated by chromatography from complex mixtures. This problem was further exacerbated by the small size of the organisms sampled in this study (typically $\ll 3 \mathrm{~cm}$ in length) and the limited material they provided. Without elaborate chemical synthesis, extensive destructive sampling, or 
establishment of large laboratory cultures to provide adequate material for compound purification, further structural elucidation and confirmation by complementary techniques (e.g. IR, NMR) is not readily possible. Thus, the structures from this study presented in Table 1 should be considered working hypotheses subject to future revision. These structures are presented to illustrate the potential diversity of haloorganic compounds present in coastal marine infauna that has not been documented previously, a result of inherent interest to marine ecologists, toxicologists and environmental chemists.

Halometabolites are widespread, but inequitably distributed among living organisms, having known roles in biosynthesis and many postulated or demonstrated ecological functions in certain taxa (Fenical 1979, Neidleman \& Geigert 1986). Specific incorporation of halogens into organic molecules in biological systems occurs via a haloperoxidase enzyme and active production of large quantities of halogenated secondary metabolites by marine plants and animals via this pathway is well known (Faulkner 1980, Fenical 1981, Gribble 1992, Butler \& Walker 1993). Infaunal taxa such as the hemichordates and the capitellid polychaetes also contain abundant ( 2 to $3 \%$ of total protein) haloperoxidase enzymes used in the biosynthesis of these molecules (Ahern et al. 1980, Chen et al. 1991, Yoon et al. 1994). In marine organisms, bromine is thought to be preferentially incorporated into organic molecules as a trade-off between its relative abundance in seawater $(\mathrm{Cl}>\mathrm{Br}>\mathrm{F}>\mathrm{I})$ and its ease of oxidation (I > Br $>\mathrm{Cl}>\mathrm{F}$ ) (Neidleman \& Geigert 1986). Recently, halogens have also been found as structural elements in small peptides and are incorporated via post-translational modification of tryptophan (Craig et al. 1997, Jimenez et al. 1997, Taylor et al. 1997).

In contrast, halogen atoms may be nonspecifically incorporated into aromatic amino acids found in structural proteins and are thought to provide stability by enhancing inter- and intra-molecular interactions among protein sheets and fibers (Hunt \& Breuer 1971). Thus, sclerotized body parts such as seta, operculum, periostricum, skeleton, and cuticle of tunicates, molluscs, polychaetes, arthropods, corals, and sponges contain these molecules (reviewed by Hunt 1984). Quinone-tanned tube structures of polychaetes also contain haloaromatic amino acids (Vovelle et al. 1994). Although the ultimate source of halometabolites from most marine organisms is not known, given the concentrations of halogenated products ( $\mu \mathrm{M}-\mathrm{mM}$; Goerke \& Weber 1990, 1991, Goerke et al. 1991, Higa et al. 1987, Woodin et al. 1993, Fielman \& Targett 1995) and haloperoxidases (Ahern et al. 1980, Chen et al. 1991, Yoon et al. 1994) and the currently observed presence of structurally diverse compounds among organisms collected from the same habitat, the most parsimonious explanation is that the worms themselves produce these compounds, unlike some sponge and bryozoan microbial associations where symbionts are the metabolite source (Unson et al. 1994, Walls et al. 1995).

In this study, possession of volatile halocompounds was not characteristic of any particular infaunal phylum or trophic mode (Table 3). However, 2 predatory taxa contained the same compounds as their prey. Glycera dibranchiata feeds on other polychaetes and contains a quantitatively and qualitatively variable mixture of compounds found in its potential prey items (Table 1, Fielman pers. obs.). The Atlantic auger, Terebra dislocata (superfamily Conacea), grazes upon the posterior end of the hemichordate Balanoglossus aurantiacus (Rupert \& Fox 1988) and contains 2,6dibromophenol, the major metabolite from posterior sections of this worm (Stewart et al. 1995). Among the Conacea, some of the cone snails (Conidae) also consume organohalogen-rich hemichordate prey (Kohn 1959). The occurrence of halogenated phenols in fish and crustaceans has been linked to their consumption of benthic prey species (Whitfield et al. 1988, 1992, 1995, Anthoni et al. 1990, Boyle et al. 1992), even though halophenols are generally toxic, causing severe metabolic and physiological disruption by depolarizing membranes and interfering with electron transport (Escher et al. 1996).

Chemical agents are often demonstrated or postulated mediators of microbial, predatory, reproductive, and recruitment processes in marine invertebrates and there is growing evidence that this is true for marine worms. For example, in the nereid polychaete Platynereis dumerilii, small, volatile, fatty-acid-derived compounds have been identified as sex pheromones (Zeeck et al. 1988, 1991). For infauna, there is evidence both for (King 1986, 1988, Giray \& King 1997a) and against (Steward et al. 1992, 1996, Yoon et al. 1994, Giray \& King 1997a, Steward \& Lovell 1997) an antimicrobial role of halogenated aromatics. There is similar disagreement as to their potential effects on meiofauna (Jensen et al. 1992, Steward et al. 1992). While polychaete worms are generally assumed to be readily consumed by predators, there is precedent for their distastefulness based on chemical grounds (McIntosh 1915 cited by George 1964, Russell 1966, Prezant 1980, Gibbs et al. 1981, Yoshiyama \& Darling 1982).

The most studied halogenated metabolites from marine infauna are the haloaromatics found in hemichordates and polychaetes (reviewed by Woodin 1991). Within these taxonomic groups, haloaromatics are found across a wide range of organism sizes (Table 3) and allocated to body parts that experience the greatest exposure to mobile epibenthic predators 
(Goerke et al. 1991, Yoon et al. 1994, Fielman \& Targett 1995). Although a recent study in Maine (USA) by Giray \& King (1997b) failed to detect an effect of halophenols on a predatory polychaete that feeds as a member of the infauna or on a hermit crab that feeds epifaunally, their study did not address epibenthic predation and its associated disruption of the sedimentary habitat by fish and large crustaceans (e.g. shrimp and blue crabs) that are arguably the critical predators shaping benthic habitats in southeastern estuarine systems (Woodin et al. 1987). Significantly, halophenols deter epibenthic predation by fish, shrimp and crabs and reduce larval recruitment in laboratory and field trials at our study sites, even at the reduced levels present in bulk sediment $\left(0.1\right.$ to $1 \mathrm{\mu g} \mathrm{ml}^{-1}$ ) (Woodin et al. 1993, 1997, unpubl., Lincoln et al. unpubl.). The ecological relevance of halocompounds from the other infauna in this report is presently unknown.

Polychaetes are an abundant source of halometabolites and often occur as the numerically dominant taxon (40 to $70 \%$ of total) in sedimentary systems (Knox 1977). Several of the haloorganic-containing polychaete species from this study have cosmopolitan distributions or form known, or suspected, widespread sibling species complexes (e.g. Mediomastus ambiseta, Heteromastus filiformis, Streblospio benedicti, and Tharyx marioni) (Knowlton 1993). These species, and others from this study, can form extensive, dense beds of individuals in many of the world's coastal benthic habitats (Hyman 1959, Gibbs 1971, Grassle \& Grassle 1974, Buhr 1976, Peterson \& Peterson 1979, Higa \& Sakemi 1983, Shaffer 1983, Levin 1984, Steward et al. 1992, Corgiat et al. 1993, Woodin et al. 1993, King et al. 1994), including haloaromatic-containing sediments surrounding pulp mills which use chlorine as a bleaching agent (Pearson \& Rosenberg 1978). Our primary message herein is to alert marine ecologists, toxicologists, and environmental chemists to the occurrence of these natural halogenated compounds in marine taxa commonly sampled in both anthropogenically impacted and pristine study sites. To explore further the significance of these observations, we are investigating the hypothesis that haloorganic compounds may partly explain macroscale benthic community composition by acting as mediators of the key ecological processes which structure these assemblages.

Acknowledgements. Field collection assistance was provided by Courtney Richmond, Mike Grove, Anne Kitchell, Jamey Watson, and Jonathan Cowart. We thank the Belle W. Baruch Institute for Marine Biology and Coastal Research for access to research sites. Support for this research was provided by EPA grant R82-4776 to D.E.L., S.A.W., C. R. Lovell and V.P. Lewis. This manuscript was improved by comments from 3 anonymous reviewers. This is contribution number 1172 of the Belle W. Baruch Institute for Marine Biology and Coastal Research.

\section{LITERATURE CITED}

Ahern TJ, Allan GG, Metcalf DG (1980) New bromoperoxidases of marine origin: partial purification and characterization. Biochim Biophys Acta 616:329-339

Anthoni U, Larsen C, Nielsen PH, Christophersen C (1990) Off-flavor from commercial crustaceans from the North Atlantic zone. Biochem Syst Ecol 18:377-379

Buhr KJ (1976) Suspension-feeding and assimilation efficiency in Lanice conchilega (Polychaeta). Mar Biol 38 $373-383$

Butler A, Walker JV (1993) Marine haloperoxidases. Chem Rev 93:1937-1944

Blood ER, Vernberg FJ (1992) Characterization of the physical, chemical and biological conditions and trends in three South Carolina estuaries. Vol II. Winyah Bay and North Inlet Estuaries. South Carolina Sea Grant Consortium, Charleston

Boyle JL, Lindsay RC, Stuiber DA (1992) Bromophenol distribution in salmon and selected seafoods of fresh- and saltwater origin. J Food Sci 57:918-922

Chadwick DJ (1990) Physical and theoretical aspects of $1 \mathrm{H}$ pyrroles. In: Jones RA (ed) Pyrroles. The synthesis and the physical and chemical aspects of the pyrrole ring. The chemistry of heterocyclic compounds. Wiley, New York, p 1-395

Chen YP, Lincoln DE, Woodin DE, Lovell CR (1991) Purification and properties of a unique flavin-containing chloroperoxidase from the capitellid polychaete Notomastus lobatus. J Biol Chem 266:23909-23915

Corgiat JM, Dobbs FC, Burger MW, Scheuer PJ (1993) Organohalogen constituents of the acorn worm Ptychodera bahamiensis. Comp Biochem Physiol 106B:83-86

Craig AG, Jimenez EC, Dykert J, Nielsen DB, Gulyas J, Abogadie FC, Porter J, Rivier JE, Cruz LJ, Olivera BM, McIntosh JM (1997) A novel post-translational modification involving bromination of tryptophan. J Biol Chem 272: $4689-4698$

Emrich R, Weyland H, Weber K (1990) 2,3,4-tribromopyrrole from the marine polychaete Polyphysia crassa. J Nat Prod 53:703-705

Escher BI, Snozzi M, Schwarzenbach RP (1996) Uptake, speciation, and uncoupling activity of substituted phenols in energy transducing membranes. Environ Sci Technol 30: $3071-3079$

Fauchald K. Jumars JA (1979) The diet of worms: a study of polychaete feeding guilds. Oceanogr Mar Biol Annu Rev 1.7:193-284

Faulkner DJ (1980) Natural organohalogen compounds. In Hutzinger $O$ (ed) The handbook of environmental chemistry. Springer-Verlag, New York, p 229-254

Fenical W (1979) Molecular aspects of halogen-based biosynthesis of marine natural products. Rec Adv Phytochem 13: $219-239$

Fenical W (1981) Natural halogenated organics. In: Duursma E, Dawson R (eds) Marine organic chemistry. Elsevier, New York, p 375-393

Fielman KT, Targett NM (1995) Variation of 2,3,4-tribromopyrrole and its sodium sulfamate salt in the hemichordate Saccoglossus kowalevskii. Mar Ecol Prog Ser 116:125-136

George JD (1964) The life history of the cirratulid worm, Cirriformia tentaculata, on an intertidal mudflat. J Mar Biol Assoc UK 44:47-65 
Gibbs PE (1971) A comparative study of reproductive cycles in four polychaete species belonging to the family Cirratulidea. J Mar Biol Assoc UK 51:745-769

Gibbs PE, Bryan GW, Ryan KP (1981) Copper accumulation by the polychaete Melinna palmata: an antipredation mechanism? J Mar Biol Assoc UK 61:707-722

Giray C, King GM (1997a) Effect of naturally occurring bromophenols on sulfate reduction and ammonia oxidation in intertidal sediments. Aquat Microb Ecol 13:295-301

Giray C, King GM (1997b) Predator deterrence and 2,4-dibromophenol conservation by the enteropneusts Saccoglossus bromophenolosus and Protoglossus graveolens. Mar Ecol Prog Ser 159:229-238

Goerke H, Weber K (1990) Locality-dependent concentrations of bromophenols in Lanice conchilega. Comp Biochem Physiol 97B:741-744

Goerke H, Weber K (1991) Bromophenols in Lanice conchilega (Polychaeta, Terebellidea): the influence of sex, weight and season. Bull Mar Sci 48:517-523

Goerke H, Emrich R, Weber K, Duchêne JC (1991) Concentrations and localization of brominated metabolites in the genus Thelepus (Polychaeta: Terebellidae). Comp Biochem Physiol 99B:203-206

Grange AH, Donnelly JR (1996) Determination of elemental compositions from mass peak profiles of the molecular ion ( $M$ ) and the $M+1$ and $M+2$ ions. Anal Chem 68 $553-560$

Grassle JF, Grassle JP (1974) Opportunistic life histories and genetic systems in marine benthic polychaetes. J Mar Res $32: 253-284$

Gribble GW (1992) Naturally occurring organohalogen compounds: a survey. J Nat PIod 55:1353-1395

Hay ME (1996) Marine chemical ecology: what's known and what's next? J Exp Mar Biol Ecol 200:103-134

Higa T, Sakemi S (1983) Environmental studies on natural halogen compounds, I. Estimation of the biomass of the acorn worm Ptychodera flava Eschscholtz (Hemichordata Enteropneusta) and excretion rate of metabolites at Kattore Bay, Kohama Island, Okinawa. J Chem Ecol 9: 495-502

Higa T, Okuda RK, Severs RM, Scheuer PJ, He CH, Changfu $X$, Clardy J (1987) Unprecedented constituents of a new species of acorn worm. Tetrahedron 43:1063-1070

Hunt $S$ (1984) Halogenated tyrosine derivatives in invertebrate scleroproteins: isolation and identification. Meth Enzymol 107:413-438

Hunt S, Breuer SW (1971) Isolation of a new naturally occurring halogenated amino acid: monochloromonobromotyrosine. Biochim Biophys Acta 252:401-404

Hyman LH (1959) The invertebrates: the smaller coelomate groups. McGraw-Hill, New York

Jensen P, Emrich R, Weber K (1992) Brominated metabolites and reduced numbers of meiofauna organisms in the burrow wall linings of the deep-sea enteropneust Stereobalanus canadensis. Deep-Sea Res 39:1247-1253

Jimenez EC, Craig AG, Watkins M, Hillyard DR, Gray WR, Gulyas J, Rivier JE, Cruz LJ, Olivera BM (1997) Bromocontryphan: post-translational bromination of tryptophan Biochemistry US 36:989-994

King GM (1986) Inhibition of microbial activity in marine sediments by a bromophenol from a hemichordate. Nature 323:257-259

King GM (1988) Dehalogenation in marine sediments containing natural sources of halophenols. Appl Environ Microbiol 54:3079-3085

King GM, Giray C, Kornfield I (1994) A new hemichordate, Saccoglossus bromophenolosus (Hemichordata: Enterop- neusta: Harrimanidae), from North America. Proc Biol Soc Wash 107:383-390

Knowlton N (1993) Sibling species in the sea. Annu Rev Ecol Syst 24:189-216

Knox GA (1977) The role of polychaetes in benthic soft-bottom communities. In: Reish DJ, Fauchald K (eds) Essays on polychaetous annelids. Allan Hancock Foundation, University of Southern California, Los Angeles, p 547-604

Kohn AJ (1959) The ecology of Conus in Hawaii. Ecol Monogr 29:47-90

Levin LA (1984) Multiple patterns of development in Streblospio benedicti Webster (Spionidae) from three coasts of North America. Biol Bull 166:494-508

McIntosh WC (1915) A monograph of the British marine annelids. Ray Society, London

Neidleman SL, Geigert J (1986) Biohalogenation: principles, basic roles, and applications. Halsted Press, New York

Paul VJ (1992) Chemical defenses of benthic marine invertebrates. In: Paul VJ (ed) Ecological roles of marine natural products. Cornell Univ Press, New York, p 164-188

Pawlik JR (1993) Marine invertebrate chemical defenses Chem Rev 93:1911-1922

Pearson TH, Rosenberg $R$ (1978) Macrobenthic succession in relation to organic enrichment and pollution of the marine environmnet. Oceanogr Mar Biol Annu Rev 16:229-311

Peterson CH, Peterson NM (1979) The ecology of intertidal flats of North Carolina: a community profile. Fish and Wildlife Service, Office of Biological Services, Slidell, LA. FWS/OBS 79/39

Prezant RS (1980) An antipredation mechanism of the polychaete Phyllodoce mucosa with notes on similar mechanisms in other potential prey. Fish Bull 77:605-616

Ruppert EE, Fox RS (1988) Seashore animals of the Southeast: a guide to common shallow-water invertebrates of the southeastern Atlantic Coast. University of South Carolina Press, Columbia, South Carolina

Russell E (1966) An investigation of the palatability of some marine invertebrates to four species of fish. Pac Sci 20: $452-460$

Shaffer PL (1983) Population ecology of Heteromastus filiformis (Polychaeta: Capitellidae). Neth J Sea Res 17(1): 106-125

Steward CC, Lovell CR (1997) Respiration and assimilation of 4-bromophenol by estuarine sediment bacteria. Microb Ecol 33:198-205

Steward CC, Pinckney J, Piceno Y, Lovell CR (1992) Bacterial numbers and activity, microalgal biomass and productivity, and meiofaunal distribution in sediments naturally contaminated with biogenic bromophenols. Mar Ecol Prog Ser 90:61-71

Steward CC, Dixon TC, Chen YP, Lovell CR (1995) Enrichment and isolation of a reductively debrominating bacterium from the burrow of a bromometabolite-producing marine hemichordate. Can J Microbiol 41:637-642

Steward CC, Nold SC, Ringelberg DB, White DC, Lovell CR (1996) Microbial biomass and community structures in the burrows of bromophenol producing and non-producing marine worms and surrounding sediments. Mar Ecol Prog Ser 133:149-165

Taghon GL, Nowell ARM, Jumars PA (1980) Induction of suspension feeding in spionid ploychaetes by high particulate fluxes. Science 210:562-564

Taylor SW, Kammerer B, Nicholson GJ, Pusecker K, Walk T, Bayer E, Scippa S, de Vincentiis M (1997) Morulin Pm: a modified polypeptide containing TOPA and 6-bromotryptophan from the morula cells of the ascidian, Phallusia mammillata. Arch Biochem Biophys 348:278-288 
Unson MD, Holland ND, Faulkner DJ (1994) A brominated secondary metabolite synthesized by the cyanobacterial symbiont of a marine sponge and accumulation of the crystalline metabolite in the sponge tissue. Mare Biol 119: $1-11$

Vovelle J, Rusaouen-Innocent $M$, Grasset $M$, Truchet $M$ (1994) Halogenation and quinone-taning of the organic tube components of some Sabellidae (Annelida: Polychaeta). Cah Biol Mar 35:441-459

Walls JT, Blackman AJ, Ritz DA (1995) Localisation of the amathamide alkaloids in surface bacteria of Amathia wilsoni Kirkpatrick, 1888 (Bryozoa: Ctenostomata). Hydrobiologia 297:163-172

Whitfield FB, Last JH, Shaw KJ, Tindale CR (1988) 2,6-dibromophenol: the cause of an iodoform-like off-flavor in some Australian crustacea. J Sci Food Agric 46:29-42

Whitfield FB, Shaw KJ, Walker Dl (1992) The source of 2,6dibromophenol: cause of an iodoform taint in Australian prawns. Water Sci Technol 25:131-138

Whitfield FB, Helidoniotis F, Svoronos D. Shaw KJ, Ford GL (1995) The source of bromophenols in some species of Australian ocean fish. Water Sci Technol 31:113-120

Woodin SA (1991) Recruitment of infauna: positive or negative cues? Am Zool 31:797-807

Woodin SA, Marinelli RL (1991) Biogenic habitat modification in marine sediments: the importance of species composi-

Editorial responsibility: Joseph Pawlik (Contributing Editor), Wilmington, North Carolina, USA tion and activity. In: Meadows PS, Tufail A (eds) The environmental impact of burrowing animals and animal burrows. Vol 63, Zool Soc London, p 231-250

Woodin SA, Walla MD, Lincoln DE (1987) Occurrence of brominated compounds in soft-bottom benthic organisms. J Exp Mar Biol Ecol 107:209-217

Woodin SA, Marinelli RL, Lincoln DE (1993) Allelochemical inhibition of recruitment in a sedimentary assemblage. J Chem Ecol 19:517-530

Woodin SA, Lindsay SM, Lincoln DE (1997) Biogenic bromophenols as negative recruitment cues. Mar Ecol Prog Ser 157:303-306

Yoon KS, Chen YP, Lovell CR, Lincoln DE, Knapp LW, Woodin SA (1994) Localization of the chloroperoxidase of the capitellid polychaete Notomastus lobatus. Biol Bull 187 $215-222$

Yoshiyama RM, Darling JDS (1982) Grazing by the intertidal fish Anoplarchus purpurescens upon a distasteful polychaete worm. Environ Biol Fish 7:39-45

Zeeck E, Hardege JD, Bartels-Hardege H, Wesselmann G (1988) Sex pheromones in a marine polychaete: determination of chemical structure. J Exp Zool 246:285-292

Zeeck E, Hardege JD, Bartels-Hardege H, Willig A, Wesselmann G (1991) Sex pheromones in a marine polychaete biologically active compounds from female Platynereis dumerilii. J Exp Biol 260:93-98

Submitted: July 20, 1998; Accepted: December 22, 1998 Proofs received from author(s): April 27, 1999 